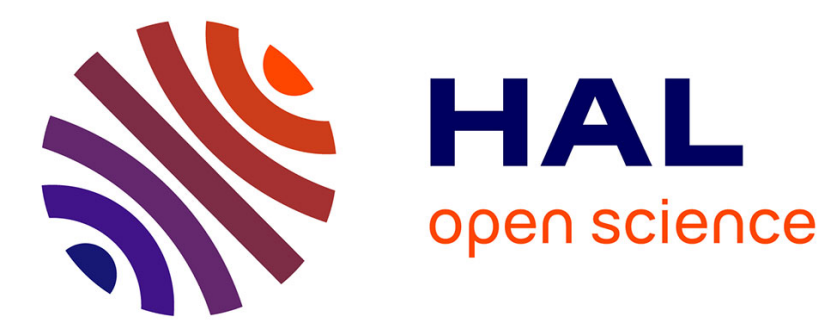

\title{
Possibilistic contextual skylines with incomplete preferences
}

\author{
Allel Hadjali, Olivier Pivert, Henri Prade
}

\section{To cite this version:}

Allel Hadjali, Olivier Pivert, Henri Prade. Possibilistic contextual skylines with incomplete preferences. 2nd International Conference on Soft Computing and Pattern Recognition (SoCPaR 2010), Dec 2010, Cergy-Pontoise, France. 10.1109/SOCPAR.2010.5686427 . inria-00534244

\section{HAL Id: inria-00534244 https://hal.inria.fr/inria-00534244}

Submitted on 21 Sep 2021

HAL is a multi-disciplinary open access archive for the deposit and dissemination of scientific research documents, whether they are published or not. The documents may come from teaching and research institutions in France or abroad, or from public or private research centers.
L'archive ouverte pluridisciplinaire HAL, est destinée au dépôt et à la diffusion de documents scientifiques de niveau recherche, publiés ou non, émanant des établissements d'enseignement et de recherche français ou étrangers, des laboratoires publics ou privés. 


\section{Possibilistic Contextual Skylines with Incomplete Preferences}

\author{
A. Hadjali \\ ENSSAT/IRISA, University of Rennes1 \\ 22305 Lannion Cedex, France \\ hadjali@enssat.fr
}

\author{
O. Pivert \\ ENSSAT/IRISA, University of Rennes1 \\ 22305 Lannion Cedex, France \\ pivert@enssat.fr
}

\author{
H. Prade \\ IRIT/CNRS, University of Toulouse 3 \\ 31062 Toulouse Cedex 09, France \\ prade@irit.fr
}

\begin{abstract}
We propose a possibility theory-based approach to the treatment of missing user preferences in skyline queries. To compensate this lack of knowledge, we show how a set of plausible preferences suitable for the current context can be derived either in a case-based reasoning manner, or using an extended possibilistic logic setting. Uncertain dominance relationships are defined in a possibilistic way and the notion of possibilistic contextual skyline is introduced. This kind of skyline allows us to return the tuples that are non-dominated with a high certainty. The paper also includes a structured overview of the different types of "fuzzy" skylines.
\end{abstract}

Keywords-Contextual preferences; skyline; possibility theory;

\section{INTRODUCTION}

In the last two decades, there has been a growing interest in preferences queries in the database community. Numerous approaches have been proposed to make database systems more flexible in supporting user preferences (see [1] for a survey). One of the most well-known approaches is that of skyline queries proposed in [2]. Skyline queries are a popular and powerful paradigm for extracting interesting objects from a multi-dimensional dataset. Given a set $r$ of $n$-dimensional tuples or points, a skyline query returns the set of non-dominated points in $r$. A tuple $t$ dominates a tuple $t^{\prime}$ if $t$ is at least as good as $t^{\prime}$ in all dimensions and strictly better than $t^{\prime}$ in at least one dimension.

Several research efforts have been made to develop efficient algorithms and to introduce different variants for skyline queries [3-7]. In particular, the problem of skyline rigidity is also addressed in [8] where a flexible dominance relationship is proposed. It allows enlarging the skyline with points that are not much dominated by any other point (even if strictly speaking they are dominated).

Moreover, in many cases, the expression of preferences inside queries may strongly depend on the context. The term 'context' refers here to any information that can be used to characterize the current query situation [9]. Recently, contextual preferences have received considerable attention in databases and information systems [10-14].

In such works, users explicitly state their preferences together with the queries according to a context. In this paper, we consider the problem of missing information in users' preferences, i.e., how to deal with skyline queries when preferences are not specified for some specific context.
To handle such a missing information situation, a set of plausible preferences based on the ones known in other contexts should be derived. In the following, possibility theory [15] is used to model the uncertainty associated with preferences. The proposed approach may be paralleled with the probabilistic method studied in [16]. We use here possibility theory due to its ability to deal with incomplete information, and its qualitative nature that makes it suitable for the problem addressed.

The paper is structured as follows. Section 2 provides a general discussion of the different ways in which a skyline may become "fuzzy" when it is refined, relaxed, simplified, extended to uncertain data, or generalized to incompletely stated context-dependent preferences. The rest of the paper focuses on this latter topic. Section 3 restates the problem using an illustrative example, recalls the probabilistic approach before motivating the approach, and discussing the notion of possibilistic contextual skyline. Section 4 details the approach on the example. Finally, Section 5 enlarges the proposed approach by discussing it in an extended possibilistic setting, before concluding.

\section{DifFERENT Types OF FuZZY SKyLINES}

There may be many different motivations for making skylines fuzzy in a way or another. First, one may want to refine the skyline by introducing some ordering between its points in order to single out the most interesting ones. Second, one may like to make it more flexible by adding points that strictly speaking do not belong to it, but are close to belonging to it. Third, one may try to simplify the skyline either by granulating the scales of the criteria, or by considering that some criteria are less important than others, or even that some criteria compensate each other, which may enable us to cluster points that are somewhat similar. Fourth, the skyline may be fuzzy due to the uncertainty or the imprecision present in the data. Lastly, the preference ordering on some criteria may depend on the context, and may be specified only for some particular or typical contexts. We now briefly review each of these ideas.

The notion of a skyline in a set of tuples is easy to state (since it amounts to exhibit non dominated points in the sense of Pareto ordering). Namely, assume we have: 
- a given a set of criteria $c_{1}, \cdots, c_{n}(n \geq 2)$ associated respectively with a set of attributes $i=1, \cdots, n$;

- a complete ordering $\succcurlyeq_{i}$ given for each criterion $i$ expressing preference between attribute values ${ }^{1}$ (the case of non comparable values is left aside).

A tuple $u=\left(u_{1}, \cdots, u_{n}\right)$ in a database $D$ belongs to the skyline $S$ if there is no other tuple $u^{\prime}=\left(u_{1}^{\prime}, \cdots, u_{n}^{\prime}\right)$ in $D$ s.t. for all $i, u_{i}^{\prime} \succcurlyeq_{i} u_{i}$ and it exists $j$ s.t. $u_{j}^{\prime} \succ_{j} u_{j}$. In other words, $\forall u^{\prime} \neq u, \exists i$ s.t. $u_{i}^{\prime} \prec_{i} u_{i}$ or $\forall j, u_{j}^{\prime} \preccurlyeq_{j} u_{j}$. Note that the case $u=u^{\prime}$ is excluded. This means in practice that if two tuples (with different names) are equal componentwise (e.g. two hotels satisfying the considered criteria exactly in the same way), they should be clustered in an equivalence class. Thus, $u$ belongs to the skyline $S$ if $u^{\prime} \neq u, \exists i$ s.t. $u_{i}^{\prime} \prec_{i} u_{i}$ (then any tuple $u^{\prime}$ is either dominated by $u$, or $u$ is non comparable w.r.t. $u^{\prime}$ ).

The first idea stated above corresponds to refine the skyline $S$ by stating that $u$ belongs to the fuzzy skyline $S_{M P}$ if $\forall u^{\prime} \neq u, \exists i$ s.t. $u_{i}$ is much preferred to $u_{i}^{\prime}$. This can be translated in fuzzy set terms by

$$
S_{M P}(u)=\min _{u^{\prime} \in D, u^{\prime} \neq u} \max _{i} M P_{i}\left(u_{i}, u_{i}^{\prime}\right)
$$

where $M P_{i}$ is a fuzzy relation expressing to what extent its first argument is much preferred to the second one for values taken in the domain of attribute $i$. Clearly, $M P_{i}\left(u_{i}, u_{i}^{\prime}\right) \leq$ $\succ_{i}\left(u_{i}, u_{i}^{\prime}\right)$ where $\succ_{i}\left(u_{i}, u_{i}^{\prime}\right)=1$ if $u_{i} \succ_{i} u_{i}^{\prime}$, and is equal to 0 otherwise. Thus, we have $S_{M P} \subseteq S$. $S_{M P}=S$ when $M P_{i}$ reduces to the crisp relation $\succ_{i}$. Clearly, $S_{M P}$ may be non normalized, or even empty.

A still more refined fuzzy skyline $S^{*}$ amounts to select those tuples, if any, that are such that $\forall u^{\prime} \neq u, \exists i$ s.t. $u_{i}$ is much preferred to $u_{i}^{\prime}$, i.e, they are in $S_{M P}$ to a high degree, and for which $\forall j \in[1, n]$ and $j \neq i$, $\nexists u^{\prime}$ s.t. $u_{j}^{\prime}$ is much preferred to $u_{j}$. Thus,

$$
\begin{aligned}
S^{*}(u)= & \min \left(\min _{u^{\prime} \in U} \max _{i} M P_{i}\left(u_{i}, u_{i}^{\prime}\right),\right. \\
& \left.\min _{j, j \neq i} \min _{u^{\prime} \in U} 1-M P_{j}\left(u_{j}^{\prime}, u_{j}\right)\right) \\
= & \min _{u^{\prime} \in U}\left(\max _{i} M P_{i}\left(u_{i}, u_{i}^{\prime}\right), \min _{j, j \neq i} 1-M P_{j}\left(u_{j}^{\prime}, u_{j}\right)\right),
\end{aligned}
$$

with $U=\left\{u^{\prime} \in D, u^{\prime} \neq u\right\}$. Clearly, $S^{*} \subseteq S_{M P}$. $S^{*}$ gathers the most interesting points, since they are much better than the others on at least one attribute, and not so bad on the other attributes (w.r.t. other existing points). To the best of our knowledge, $S^{*}$ and $S_{M P}$ do not seem to have been considered in the literature.

Rather than refining the skyline, a second type of fuzzy skyline corresponds to the idea of relaxing it, i.e., $u$ still belongs to the skyline to some extent (but to a less extent), if $u$ is only weakly dominated by any other $u^{\prime}$. Note first that this does not amount to weakening the requirement $\exists i$ s.t. $u_{i}^{\prime} \prec_{i} u_{i}$, replacing $\prec_{i}$ by $\max \left(\prec_{i}, E_{i}\right)$ where $E_{i}$ is an approximate indifference relation, as $\succ_{i}$ has been

${ }^{1} u \succ v$ means $u$ is preferred to $v . u \succcurlyeq v$ means $u$ is at least as good as $v$, i.e., $u \succcurlyeq v \Leftrightarrow u \succ v \vee u \approx v$, where $\approx$ denotes indifference. strengthened for defining $S_{M P}$. Indeed, this would allow for cases where $\exists i$ s.t. $u_{i}^{\prime}$ and $u_{i}$ are approximately equally preferred, while $u^{\prime}$ dominates $u$ on the rest of the attributes. Rather, such a relaxed skyline $S_{R E L}$ can be defined as the extent $S_{R E L}(u)$ to which it is false that there exists a tuple $u^{\prime}$ much preferred to $u$ w.r.t. all attributes (this expression was proposed in [8])

$$
\begin{aligned}
S_{R E L}(u) & =1-\max _{u^{\prime} \in U} \min _{i} M P_{i}\left(u_{i}^{\prime}, u_{i}\right) \\
& =\min _{u^{\prime} \in U} \max _{i}\left(1-M P_{i}\left(u_{i}^{\prime}, u_{i}\right)\right) .
\end{aligned}
$$

Note the formal similarity with the expression of $S_{M P}$, changing $M P_{i}\left(u_{i}, u_{i}^{\prime}\right)$ into $1-M P_{i}\left(u_{i}^{\prime}, u_{i}\right)$, but mind that $u_{i}^{\prime}$ and $u_{i}$ are interchanged. Clearly, $S \subseteq S_{R E L}$, since $1-$ $M P_{i}\left(u_{i}^{\prime}, u_{i}\right) \geq \preccurlyeq_{i}\left(u_{i}^{\prime}, u_{i}\right)$. If $u \in S, S_{R E L}(u)=1$.

Another way of relaxing $S$ is to consider that $u$ still belongs to a fuzzily extended skyline $S_{F E}$ if $u$ is close to $u^{\prime}$ with $u^{\prime} \in S$. This would lead to the following definition

$$
S_{F E}(u)=\max _{u^{\prime} \in D, u^{\prime} \neq u} \min \left(S\left(u^{\prime}\right), \min _{i} E_{i}\left(u_{i}, u_{i}^{\prime}\right)\right)
$$

where $S\left(u^{\prime}\right)=1$ if $u^{\prime} \in S$ and 0 otherwise. $S_{F E}(u)=$ 1 if $u \in S$ also. Still, one may have $S_{F E}(u)>0$ while $S_{R E L}(u)=0$. Indeed, a point may be close to a skyline point while being much dominated by another skyline point (e.g. in case of two criteria, a pair $(x, y)$ may be close to a skyline point $\left(x^{\prime}, y\right)$ if $x$ is less preferred than $x^{\prime}$ but close to it, while there exists $\left(x, y^{\prime}\right)$ where $y^{\prime}$ is much preferred to $y$ ). Such a situation corresponds to a lack of local "smoothness" of the skyline. So, $S_{F E}$ may be found too permissive.

On the contrary, it may be desirable to simplify the skyline just because it contains too many points $[3,4]$. There are many ways to do it. The definitions of $S^{*}$ and $S_{M P}$ serve this purpose, but they may be empty as already said. We now briefly mention three different ways to simplify the skyline, which are also meaningful. First, one may consider that the set of criteria is partitioned in subsets of decreasing importance. Then we may judge that a tuple cannot belong to the skyline only because it strictly dominates all the other tuples on a non fully important criterion. Indeed it may look strange that a tuple belongs to the skyline while it is dominated on all the important criteria, even if its value on a secondary criterion makes the tuple finally incomparable. In this view, less important criteria may be only used to get rid of tuples that are dominated on immediately less important criteria, in case of ties on more important criteria. Note that however the importance of a criterion might be changed in case of a very strong (and very rare) dominance for a non fully important criterion.

A second, completely different idea for simplifying a skyline is to use coarser scales for the evaluation of the attributes (e.g., moving from precise values to rounded values). This will lead to more comparable (or even identical) tuples. Still another way to increase the number of comparable tuples is to use a 2-discrimin (or more generally an order k-discrimin) ordering [17]. This can be illustrated 
by the following example, where we compare hotels on the basis of their price, distance to the station, and distance to a conference location. Then $(80,1,3)$ et $(70,3,1)$ are not Pareto comparable, while we may consider that the two distance criteria play similar roles and that there is equivalence between the sub-tuples $(1,3)$ and $(3,1)$ leading to compare the tuples on the remaining components. Order $k$-discrimin will allow for equivalences between sub-tuples of size $k$ or less.

The fourth type of "fuzzy" skyline is quite clear. When attributes values are imprecisely or more generally fuzzily known, we are led to define the tuples that certainly belong to the skyline, and those which only possibly belong to the skyline, using necessity and possibility measures [7]. In the following we concentrate on the last category of "fuzzy" skyline that is induced by an incompletely known contextdependency of the involved preferences.

\section{UNCERTAIN CONTEXTUAL SKYLINES}

\section{A. A Motivating Example}

We use an example taken from [16]. It consists of a relation with 3 attributes Price, Distance and Amenity about a set of hotels (Table I). For instance, a skyline query returns "those hotels for which there is no cheaper and, at the same time, closer to the beach alternative". One can easily

Table I: Relation describing hotels

\begin{tabular}{|c|c|c|c|}
\hline Hotel & Price & Distance & Amenity \\
\hline$h_{1}$ & 200 & 10 & Pool(P) \\
\hline$h_{2}$ & 300 & 10 & Spa(S) \\
\hline$h_{3}$ & 400 & 15 & Internet'I) \\
\hline$h_{4}$ & 200 & 5 & Gym(G) \\
\hline$h_{5}$ & 100 & 20 & Internet(I) \\
\hline
\end{tabular}

check that the skyline contains hotels $h_{4}$ and $h_{5}$. In other terms, hotels $h_{4}$ and $h_{5}$ represent non-dominated hotels w.r.t. Price and Distance dimensions. In many cases, results of

Table II: Contextual Skylines

\begin{tabular}{|c|c|c|}
\hline Context & Preferences & Skyline \\
\hline$C_{1}$ : Business, June & $I \succ G, I \succ\{P, S\}$ & $h_{3}, h_{4}, h_{5}$ \\
& $G \succ P, S$ & \\
\hline$C_{2}$ : Vacation & $S \succ\{P, I, G\}$ & $h_{2}, h_{4}, h_{5}$ \\
\hline$C_{3}$ : Summer & $P \succ\{I, G\}$ & $h_{1}, h_{2}, h_{4}, h_{5}$ \\
& $S \succ\{I, G\}$ & \\
\hline$C_{q}$ : Business, Summer & - & $?$ \\
\hline
\end{tabular}

preference queries may depend on the context. To this end, we consider contextual skyline queries that require that the dominance relationships will be defined relative to a context. To illustrate this idea, consider three contexts $C_{1}, C_{2}$ and $C_{3}$ shown in Table II (where a given context can be composed at most by two context parameters (Purpose, Period)). For example, when the user is on a business trip in June (context $C_{1}$ ), hotels $h_{3}, h_{4}$ and $h_{5}$ are the results of the skyline query for $C_{1}$. See Table II for contexts $C_{2}$ and $C_{3}$ and their corresponding skylines.
Let us now examine situation $C_{q}$ (fourth row in Table II), where the user plans a business trip in the summer but states no preferences. To understand the uncertainty present in preferences in the context $C_{q}$, let us consider amenities Internet (I) and Pool (P). One can observe that: (i) $I$ may be preferred to $P$ as in $C_{1}$; (ii) $P$ may be preferred to $I$ as in $C_{3}$, or (iii) $I$ and $P$ may be equally favorable as in $C_{2}$. In fact, all three alternatives hold with some plausibility that depends on the similarity of $C_{q}$ to $C_{1}, C_{2}$, and $C_{3}$. Moreover, the uncertainty propagates to the dominance relationships, i.e., every hotel may dominate another with a certainty degree that depends on the context.

\section{B. Contextual skylines}

Let $D$ be a relational database with attributes $A_{1}, \cdots, A_{n}$. Let $C$ be a context associated with the user query. $C$ is modeled as a set of parameter-value pairs. E.g., context $C_{1}$ of Table II is expressed as Purpose $=$ Business, Period $=$ June. Let $\operatorname{dom}\left(A_{j}\right)$ be the domain of attribute $A_{j}$ and $u, v \in \operatorname{dom}\left(A_{j}\right)$. We use the notation $u \succ_{A_{j}} v$ to express that $u$ is preferred to $v$ for attribute $A_{j}$. This implies that for two database tuples $t$ and $t^{\prime}$, if $t . A_{j}=u, t^{\prime} \cdot A_{j}=v, u \succ_{A_{j}} v$, and $t . A_{k} \approx t^{\prime} . A_{k}$ for all $k \neq j$, then $t$ is preferred to $t^{\prime}$. Now, if preferences depend on context, we denote by $u \succ_{A_{j}} v \mid C_{i}$ a preference on attribute $A_{j}$ that holds for a particular context $C_{i}$.

Example (Cont'). Let us come back to our previous example and consider the Amenity attribute (denoted by $A$ ). The following contextual preferences hold (see Table II): $I \succ_{A} G\left|C_{1}, I \succ_{A} P\right| C_{1}, I \succ_{A} S\left|C_{1}, G \succ_{A} P\right| C_{1}$ and $G \succ_{A} S \mid C_{1}$. One can observe that the set of preferences for attribute $A_{j}$ in context $C_{i}$ defines a (strict) partial order. The Contextual Skyline Query (CSQ) returns the database tuples that are not dominated by any other in context $C_{i}$. Let $t$ and $t^{\prime}$ two tuples, $t$ dominates $t^{\prime}$ in $C_{i}$, noted $t \succ_{A} t^{\prime} \mid C_{i}$,

$$
\text { iff } \forall j, t . A_{j} \succcurlyeq t^{\prime} \cdot A_{j} \mid C_{i} \text { and } \exists k, t . A_{k} \succ t^{\prime} \cdot A_{k} \mid C_{i} \text {. }
$$

\section{Problem Description}

Let the profile of a user refer to the set of contextual preferences specified in the past. Now, given the current context $C_{q}$ for which preferences are missing, and given the user's profile, the problem is how to determine the nondominated tuples in context $C_{q}$.

In the case where $C_{q}$ perfectly matches only one of the contexts included in the profile, the problem reduces to a precise CSQ. The difficulty arises when $C_{q}$ is not present in the profile. In this case, what should the user's preferences be? A solution is to interpolate them on the basis of the profile. This entails that derived preferences will be pervaded with uncertainty, i.e., for two tuples $t$ and $t^{\prime}$, the relation $t$ dominates $t^{\prime}$ holds only with some plausibility degree.

In [16] an approach is proposed where uncertainty is modeled using probability theory. The probability that $t$ 
dominates $t^{\prime}$ in context $C_{q}$ is defined as ${ }^{2} \operatorname{Pr}\left(t \succ t^{\prime}\right.$ $\left.C_{q}\right)=\prod_{j} \operatorname{Pr}\left(t \cdot A_{j} \succcurlyeq_{A_{j}} t^{\prime} \cdot A_{j} \mid C_{q}\right)$ if $t \neq t^{\prime}$ and 0 otherwise, with $\operatorname{Pr}\left(u \succ_{A_{j}} v \mid C_{q}\right)=\sum_{k} \operatorname{Sim}\left(C_{q}, C_{k}\right)$. $\left|u \succ_{A_{j}} \quad v\right| C_{k} \mid / \sum_{k} \operatorname{Sim}\left(C_{q}, C_{k}\right)$ and $u, v \in \operatorname{dom}\left(A_{j}\right)$. $\operatorname{Sim}\left(C_{q}, C_{k}\right)$ is the similarity between the contexts $C_{q}$ and $C_{k}$ and $\left|u \succ_{A_{j}} v\right| C_{k} \mid$ is 1 if such a preference exists, and 0 otherwise. The probability that a tuple $t$ belongs to the skyline is also defined, i.e., $P_{s k y}^{C_{i}}=\prod_{t^{\prime} \neq t}\left(1-\operatorname{Pr}\left(t^{\prime} \succ\right.\right.$ $\left.\left.t \mid C_{i}\right)\right)$. The major drawback of this approach stems from the fact that the probabilistic assessment of uncertainty should be quantitative, and is hence more demanding in terms of data. In the following, we propose an alternative approach that relies on possibility theory where uncertainty modeling can be qualitative, thus avoiding the need of quantifying uncertainty if information is poor.

\section{Possibilistic Contextual Skylines}

Here, the possibilistic framework (see Appendix) is used to model uncertainty. To achieve this, each uncertain contextual preference will be associated with a certainty (necessity) degree. We use the following notation:

$$
N\left(u \succcurlyeq_{A_{j}} v \mid C_{i}\right)=\alpha
$$

with $\alpha \in[0,1]$. This expression reads: in context $C_{i}, u$ is preferred or equally preferred to $v$ with a certainty $\alpha$. Since the degree $\alpha$ plays an ordinal role only, the interval $[0,1]$ can be replaced by any linearly ordered scale. Using the dual relation between necessity measure and possibility measure $\Pi$, the following relation holds

$$
\Pi\left(\neg\left(u \succcurlyeq_{A_{j}} v \mid C_{i}\right)\right)=1-\alpha
$$

The approach proposed relies on a well-known CaseBased Reasoning (CBR) principle [18] which states that "the more similar the values of two situations for attributes $s$ and $s_{0}$, the more possible the similarity of their values on their outcome attributes $t$ and $t_{0}$ ". Thus, for each pair $(u, v) \in \operatorname{dom}\left(A_{j}\right) \times \operatorname{dom}\left(A_{j}\right)$, one can assess the possibility that the relation $u \succ_{A_{j}} v \mid C_{q}$ holds in the following way:

$$
\Pi\left(u \succ_{A_{j}} v \mid C_{q}\right)=\max _{i}\left(\min \left(\operatorname{Sim}\left(C_{q}, C_{i}\right), N\left(u \succ_{A_{j}} v \mid C_{i}\right)\right)\right)
$$

where $\operatorname{Sim}\left(C_{q}, C_{i}\right)$ is detailed further and $N\left(u \succ_{A_{j}} v \mid C_{i}\right)$ is the certainty that $u \succ_{A_{j}} v$ holds in the context $C_{i}$ (i.e., $N\left(u \succ_{A_{j}} v \mid C_{i}\right)=1$ if $u \succ_{A_{j}} v$ is true, and 0 otherwise).

Note that formula (3), which parallels the probabilistic expression, is in fact an empirical adaptation of the above principle. When used in CBR, the principle amounts to the expression of a possibility as a maximum of possibility degrees weighted by similarities. Note that: (i) When $\operatorname{Sim}\left(C_{q}, C_{i}\right)>0, \Pi\left(u \succ_{A_{j}} v \mid C_{q}\right)=0$ if there is no certainty at all that $u \succ_{A_{j}} v \mid C_{i}$ holds (i.e. $N\left(u \succ_{A_{j}} v \mid C_{i}\right)=0$ ), and $\Pi\left(u \succ_{A_{j}} v \mid C_{q}\right)=1$ if it is certain that $u \succ_{A_{j}} v \mid C_{i}$ holds (i.e., $N\left(u \succ_{A_{j}} v \mid C_{i}\right)=1$ ) when $C_{q}$ matches

\footnotetext{
${ }^{2}$ In [16], $u \succcurlyeq v$ is defined as $u \succ v \vee u=v$.
}

exactly one $C_{i}$; (ii) Now, if it is certain that $u \succ_{A_{j}} v \mid C_{i}$ holds in all contexts, one has only $\Pi\left(u \succ_{A_{j}} v \mid C_{q}\right)=$ $\max _{i} \operatorname{Sim}\left(C_{q}, C_{i}\right)$.

In a similar way as in (3), one can compute the possibility that the relation $\left(u \succ_{A_{j}} v \mid C_{q}\right)$ does not hold, i.e.,

$$
\begin{gathered}
\Pi\left(\neg\left(u \succ_{A_{j}} v \mid C_{q}\right)\right)=\max _{i}\left(\operatorname { m i n } \left(\operatorname{Sim}\left(C_{q}, C_{i}\right),\right.\right. \\
\left.\left.N\left(\neg\left(u \succ_{A_{j}} v \mid C_{i}\right)\right)\right)\right) .
\end{gathered}
$$

Uncertain dominance relationships. Since preferences are pervaded with uncertainty, the dominance relationship becomes uncertain. Then, for two tuples $t$ and $t^{\prime}$, the certainty degree that $t$ dominates $t^{\prime}$ in the context $C_{i}$ is now defined as:

$$
N\left(t \succ t^{\prime} \mid C_{i}\right)=\left\{\begin{array}{l}
0 \text { if } \forall j, t . A_{j} \approx t^{\prime} \cdot A_{j} \mid C_{i} \\
\min _{j} N\left(t . A_{j} \succcurlyeq_{A_{j}} t^{\prime} . A_{j} \mid C_{i}\right) \text { otherwise }
\end{array}\right.
$$

where the second case applies only when tuples do not have equally preferred values in all attributes of interest.

Classically, a tuple is in the skyline if it is not dominated by any other tuple of the same relation. To adapt this definition to our case, one can compute the skyline certainty of a tuple $\mathrm{t}$ in the following way:

$N_{s k y}^{C_{i}}(t)=\min _{t^{\prime} \neq t} N\left(\neg\left(t^{\prime} \succ t\right) \mid C_{i}\right)=\min _{t^{\prime} \neq t}\left\{1-\Pi\left(t^{\prime} \succ t \mid C_{i}\right)\right\}$

Formula (5) expresses the certainty that a tuple $t$ is not dominated by any other tuple $t^{\prime}$ of the same relation. One can easily check that in the deterministic case, when all preferences are totally certain, the scalar $N_{s k y}^{C_{i}}(t)$ is equal to

- 1 if $t$ is not dominated, i.e., $\forall t^{\prime}, N\left(\neg\left(t^{\prime} \succ t\right) \mid C_{i}\right)=1$, - 0 otherwise, i.e., $\exists t^{\prime}, N\left(\neg\left(t^{\prime} \succ t\right) \mid C_{i}\right)=0$.

$\alpha$-Certain Contextual Skyline Query. Given a database $D$ and a set of uncertain preferences, our final aim is to retrieve the tuples $\mathrm{t}$ whose skyline certainty is greater or equal to a user-defined threshold $\alpha$, i.e., compute the set $\alpha-C C S Q\left(D / C_{i}\right)$ defined as:

$$
\alpha-C C S Q\left(D / C_{i}\right)=\left\{t \in D, N_{s k y}^{C_{i}}(t) \geq \alpha\right\}
$$

Context Similarity. Let us now say a few words about the similarity relation, Sim, between contexts. Let us consider that each context $C$ contains $n$ context parameters $\left(P_{1}, P_{2}, \ldots, P_{n}\right)$ and assume also that for each parameter $P_{i}$ there exists a function $\operatorname{Sim}_{P_{i}}$ that measures the similarity between two values of its domain $\operatorname{dom}\left(P_{i}\right)$. One may use the following functions depending on the domain type: (i) for numerical domains, $\operatorname{Sim}_{P_{i}}(x, y)=1-(\mid x-$ $y \mid /(M-m))$ where $M$ (resp. $m$ ) is the maximum (resp. minimum) value of $\operatorname{dom}\left(P_{i}\right)$; (ii) for categorical domains, $\operatorname{Sim}_{P_{i}}(x, y)=|\operatorname{lvs}(x) \cap \operatorname{lvs}(y)| /|\operatorname{lvs}(x) \cup \operatorname{lvs}(y)|$, i.e., the Jaccard index where $\operatorname{lvs}(x)$ stands for the set of leaves under $x$; (iii) for nominal domains, $\operatorname{Sim}_{P_{i}}(x, y)=1$ if $x=y, 0$ otherwise. The similarity measure between two contexts $C$ and $C^{\prime}$ can be defined as: $\operatorname{Sim}\left(C, C^{\prime}\right)=$ $\min _{k=1, n} \operatorname{Sim}_{P_{k}}\left(C . P_{k}, C^{\prime} . P_{k}\right)$. 


\section{A Detailed Example}

We use the example provided in Section III to illustrate the notions described in the previous section. Let us first estimate the extent to which the query context $C_{q}$ is similar to the contexts present in the user's profile. Assume that the following similarity relations between parameter values are available: $\operatorname{Sim}_{\text {Purpose }}($ Business, Vacation $)=0$, $\operatorname{Sim}_{\text {Period }}($ June, Summer $)=1 / 3$. Then, $\operatorname{Sim}\left(C_{q}, C_{1}\right)=$ $1 / 3, \operatorname{Sim}\left(C_{q}, C_{2}\right)=0$ and $\operatorname{Sim}\left(C_{q}, C_{3}\right)=1$.

Now based on formula (3), uncertain preferences in the context $C_{q}$ can be inferred and are summarized in Table III. Let us provide some details about the calculus of one of the

Table III: Preferences Possibilities $\Pi\left(u \succ_{A} v \mid C_{q}\right)$

\begin{tabular}{|c|c|c|c|c|}
\hline$u \backslash v$ & $\mathrm{I}$ & $\mathrm{G}$ & $\mathrm{P}$ & $\mathrm{S}$ \\
\hline $\mathrm{I}$ & - & $1 / 3$ & $1 / 3$ & $1 / 3$ \\
\hline $\mathrm{G}$ & 0 & - & $1 / 3$ & $1 / 3$ \\
\hline $\mathrm{P}$ & 1 & 1 & - & 0 \\
\hline $\mathrm{S}$ & 1 & 1 & 0 & - \\
\hline
\end{tabular}

degrees of possibility given in Table III: $\Pi\left(I \succ_{A} G \mid C_{q}\right)=$ $\max \left(\min \left(\operatorname{Sim}\left(C_{q}, C_{1}\right), N\left(I \succ_{A} G \mid C_{1}\right)\right), \min \left(\operatorname{Sim}\left(C_{q}\right.\right.\right.$, $\left.\left.\left.C_{2}\right), N\left(I \succ_{A} G \mid C_{2}\right)\right), \min \left(\operatorname{Sim}\left(C_{q}, C_{3}\right), N\left(I \succ_{A} G \mid C_{3}\right)\right)\right)$ $=\max (\min (1 / 3,1), \min (0,0), \min (1,0))=1 / 3$.

To assess the skyline certainty of each tuple $t$ of Table I, we need to compute the uncertain dominance relationships among tuples shown in this table. See Table IV for the skyline certainties obtained for all tuples. Let us take an example to illustrate the calculus of dominance certainties of Table IV.

Table IV: Dominance certainties $N\left(s \succ t \mid C_{q}\right)$

\begin{tabular}{|c|c|c|c|c|c|}
\hline$s \backslash t$ & $h_{1}$ & $h_{2}$ & $h_{3}$ & $h_{4}$ & $h_{5}$ \\
\hline$h_{1}$ & 0 & 0 & $2 / 3$ & 0 & 0 \\
\hline$h_{2}$ & 0 & 0 & $2 / 3$ & 0 & 0 \\
\hline$h_{3}$ & 0 & 0 & 0 & 0 & 0 \\
\hline$h_{4}$ & 0 & 0 & 0 & 0 & 0 \\
\hline$h_{5}$ & 0 & 0 & 0 & 0 & 0 \\
\hline
\end{tabular}

$N\left(h_{1} \succ h_{3} \mid C_{q}\right)=\min _{j} N\left(h_{1} \cdot A j \succcurlyeq h_{3} \cdot A j \mid C_{q}\right)=$ $\min \left(N\left(200 \succcurlyeq 400 \mid C_{q}\right), N\left(10 \succcurlyeq 15 \mid C_{q}\right), N\left(P \succcurlyeq I \mid C_{q}\right)\right)$. One can easily see that $N\left(200 \succcurlyeq 400 \mid C_{q}\right)=$ $N\left(10 \succcurlyeq 15 \mid C_{q}\right)=1$. By $N(A \cup B)=\max (N(A), N(B))$, we have $N\left(P \succcurlyeq I \mid C_{q}\right)=\max (N(P \succ$ $\left.\left.I \mid C_{q}\right), N\left(P \approx I \mid C_{q}\right)\right)=N\left(P \succ I \mid C_{q}\right)$ since $N\left(P \approx I \mid C_{q}\right)=0$. Using formula (3'), $\Pi\left(\neg\left(P \succ I \mid C_{q}\right)\right)=$ $\max (\min (1 / 3,1), \min (0,1), \min (1,0))=1 / 3$. This means that $N\left(P \succ I \mid C_{q}\right)=2 / 3$ and $N\left(P \succcurlyeq I \mid C_{q}\right)=2 / 3$. Thus, $N\left(h_{1} \succ h_{3} \mid C_{q}\right)=\min [1,1,2 / 3]=2 / 3$.

We have now to compute the skyline certainty for each tuple $t$, i.e., $N_{s k y}^{C_{q}}(t)$. To achieve this by applying formula (5), we need the degree of certainty that $t$ is not dominated by another tuple $s$ (i.e., $N(\neg(s \succ t \mid C q))$ ). Table $\mathrm{V}$ summarizes those certainty degrees. One can observe that $h_{1}$ is not dominated by $h_{2}$ for sure (i.e., $N\left(\neg\left(h_{2} \succ\right.\right.$ $\left.h_{1}(C q)\right)=1$ ), while $h_{1}$ is not dominated by $h_{4}$ with a certainty equal to $2 / 3$. Let us give details about the calculus of $N\left(\neg\left(h_{2} \succ h_{1} \mid C q\right)\right)$. It is easy to see that $N\left(\neg\left(h_{2} \succ h_{1} \mid C_{q}\right)\right)=1-\Pi\left(h_{2} \succ h_{1} \mid C_{q}\right)$. Unfortunately from Table IV, no information can be obtained on $\Pi\left(h_{2} \succ h_{1} \mid C_{q}\right)$ (we have only $\Pi\left(\neg\left(h_{2} \succ h_{1} \mid C_{q}\right)\right)=1$ due to $\left.N\left(h_{2} \succ h_{1} \mid C q\right)=0\right)$. It is why, we use the following formula: $\Pi\left(h_{2} \succ h_{1} \mid C_{q}\right)=\Pi\left(\wedge_{j}\left(h_{2} \cdot A_{j} \succcurlyeq h_{1} \cdot A_{j}\right) \mid C_{q}\right)=$ $\min _{j} \Pi\left(h_{2} \cdot A_{j} \succcurlyeq h_{1} \cdot A_{j} \mid C_{q}\right)=\min (0,1,0)=0$ (see Appendix). So, $N\left(\neg\left(h_{2} \succ h_{1} \mid C_{q}\right)\right)=1$. In a similar way, we compute $N\left(\neg\left(h_{4} \succ h_{1} \mid C_{q}\right)\right)=1-\Pi\left(h_{4} \succ\right.$ $\left.h_{1} \mid C_{q}\right)=1-\min (1,1,1 / 3)=2 / 3$. From Table IV and using the relation $\min \left(N(A), N\left(A^{c}\right)\right)=0$, we have $N\left(\neg\left(h_{1} \succ h_{3} \mid C_{q}\right)\right)=0$.

Table V: Non-dominance certainties $N\left(\neg\left(s \succ t \mid C_{q}\right)\right)$

\begin{tabular}{|c|c|c|c|c|c|}
\hline$s \backslash t$ & $h_{1}$ & $h_{2}$ & $h_{3}$ & $h_{4}$ & $h_{5}$ \\
\hline$h_{1}$ & 1 & 1 & 0 & 1 & 1 \\
\hline$h_{2}$ & 1 & 1 & 0 & 1 & 1 \\
\hline$h_{3}$ & 1 & 1 & 1 & 1 & 1 \\
\hline$h_{4}$ & $2 / 3$ & $2 / 3$ & 1 & 1 & 1 \\
\hline$h_{5}$ & 1 & 1 & 1 & 1 & 1 \\
\hline$N_{s k y}^{C_{q}}(t)$ & $2 / 3$ & $2 / 3$ & 0 & 1 & 1 \\
\hline
\end{tabular}

According to formula (5), one can compute the skyline certainty of each hotel $h_{i}$ of Table I in context $C_{q}$, see the last row of Table V. For instance, we have $N_{s k y}^{C_{q}}\left(h_{1}\right)=$ $2 / 3$ and $N_{s k y}^{C_{q}}\left(h_{4}\right)=1$. So, $\alpha-\operatorname{CCSQ}\left(D / C_{q}\right)=$ $\left\{h_{1}, h_{2}, h_{4}, h_{5}\right\}$ for $\alpha=2 / 3$. Let us take a look at hotel $h_{3}$ which belongs to the skyline with a certainty 0 despite the fact that context $C_{q}$ is somewhat similar to context $C_{1}$ (where $h_{3}$ is in the skyline). This can be argued by the fact that $C_{q}$ is more similar to $C_{3}$ than to $C_{1}$, and our approach leads to a result which is more similar to the one provided in $C_{3}$ (where $h_{3}$ is not in the skyline) than in $C_{1}$. However, one could check that $h_{3}$ belongs to the skyline with a possibility degree equal to $1 / 3$. Note that the degree $1 / 3$ have to be interpreted ordinally and not quantitatively.

\section{TOWARds a Possibilistic Logic Handling}

In the previous sections, we have proposed a similaritybased handling of context-dependent preferences. Still, such an approach is not fully satisfactory in different situations. First, the context $C_{q}$ may be in fact a subcontext of one (or several) contexts where the preference orderings is/are known. Then, determining the preferences applicable to $C_{q}$ is a simple matter of logical inference, not of CBR-like reasoning. Moreover, it may happen that among the contexts where the preferences are known, we have two contexts $\mathrm{C}$ and $C^{\prime}$ such that $C^{\prime} \vDash C$ (i.e., $C^{\prime}$ entails $C$ ), with conflicting preferences. This has to be interpreted in a non monotonic logic manner, considering that we use the C-preferences if $C_{q} \vDash C$ and $C_{q} \not \models C^{\prime}$, and the $C^{\prime}$-preferences if $C_{q} \vDash C^{\prime}$. This calls for a logical approach that is now outlined.

Generally, context dependency can be handled in the setting of an extended possibilistic logic, as the one used in [19] for preference queries with uncertain data. Suppose 
we have one criterion like amenity in the example, with values including $a$ and $b$ (e.g., internet and pool). Using possibilistic logic, the preference of $a$ over $b$ in context $C_{1}$ writes $\left\{\left(\neg C_{1} \vee \neg a(x) \vee A n s(x), 1, \mathbf{1}\right),\left(\neg C_{1} \vee \neg b(x) \vee\right.\right.$ Ans $(x), \alpha, \mathbf{1})\}$, where $a(x)$ expresses that $a$ is true for item $x$ and $\operatorname{Ans}(x)$ that item $x$ is in the set of eligible answers, and $\alpha$ is a priority level, and $\mathbf{1}$ expresses the (full) certainty that e.g., having $b$ in $C_{1}$ has a priority level $\alpha<1$, while the preference of $b$ over $a$ in context $C_{2}$ writes $\left\{\left(\neg C_{2} \vee\right.\right.$ $\left.\neg b(x) \vee \operatorname{Ans}(x), 1, \mathbf{1}),\left(\neg C_{2} \vee \neg a(x) \vee \operatorname{Ans}(x), \alpha, \mathbf{1}\right)\right\}$.

Suppose both $C^{\prime}$ and $C_{2}$ are true, where $C^{\prime}$ is a subcontext of $C_{1}$, which writes $\left(\neg C^{\prime} \vee C_{1}, 1, \mathbf{1}\right)$, while 1 is the maximal priority. Then we can deduce $(\neg a(x) \vee A n s(x), 1, \mathbf{1})$ and $(\neg b(x) \vee A n s(x), 1, \mathbf{1})$ by applying the possibilistic resolution rule both on the priority levels and on the certainty levels. Thus opposite preferences are understood disjunctively: here having $a$ is as good as having $b$.

Assume further, e.g., that we know $a\left(h_{1}\right)$, i.e. hotel $h_{1}$ has amenity $a$. Then, if we just know we are in context $C^{\prime}$, we can conclude $\left(A n s\left(h_{1}\right), 1, \mathbf{1}\right)$, which means that $h_{1}$ is non-dominated on the Amenity attribute (due to its maximal priority), with full certainty (1). Lastly, if we want to also handle similar contexts, we may here express the counterpart of the CBR principle by stating that if the formula $(\neg C \vee \neg g(x) \vee A n s(x), \lambda, \boldsymbol{\rho})$ holds, then the formula $\left(\neg C^{\prime} \vee \neg g(x) \vee \operatorname{Ans}(x), \lambda, \min \left(\rho, \boldsymbol{S i m}\left(\boldsymbol{C}, \boldsymbol{C}^{\prime}\right)\right)\right)$ also holds. This expresses that we downgrade the certainty of a preference by the amount of similarity between the contexts.

\section{CONCLUSION}

The paper has provided a structured discussion of the different types of "fuzzy" skylines that may be imagined, before focusing on the case of incompletely specified user preferences in specific contexts. We have shown how to derive a set of plausible preferences suitable for the context at hand on the basis of the information known for other contexts. We use a possibilistic setting for defining uncertain dominance relationships. Then the user is provided with the tuples that are not dominated with a high certainty, leading to a notion of possibilistic contextual skyline.

\section{ApPendiX. Brief Overview ON POSSIBLITy Theory}

Possibility theory [15] offers a qualitative model for uncertainty where any event $A$ is characterized by two measures: its possibility $\Pi$ (expressing the fact that $A$ may more or less occur) and its necessity $N$ (expressing that $A$ will occur more or less for sure). The necessity $N$ of $A$ is defined as: $N(A)=1-\Pi\left(A^{c}\right)$ where $A^{c}$ is the event opposite to $A$. The following properties are of interest: (i) $\max \left(\Pi(A), \Pi\left(A^{c}\right)\right)=1$; (ii) $\Pi(A \cup B)=$ $\max (\Pi(A), \Pi(B))$; (iii) $\Pi(A)<1 \Rightarrow N(A)=0$; (iv) $N(A \cap B)=\min (N(A), N(B))$; (v) $\Pi(A \cap B)=$ $\min (\Pi(A), \Pi(B))$ and $N(A \cup B)=\max (N(A), N(B))$, if $A$ and $B$ are logically independent.
Possibilistic logic is based on the inference rule:

$N(p \vee q) \geq \alpha$ and $N(\neg p \vee r) \geq \beta \Rightarrow N(q \vee r) \geq \min (\alpha, \beta)$.

\section{REFERENCES}

[1] A. Hadjali, S. Kaci, and H. Prade, "Database preferences queries: a possibilistic logic approach with symbolic priorities," in Proc. FoIKS, 2008, pp. 291-310.

[2] S. Borzsony, D. Kossmann, and K. Stocker, "The skyline operator," in Proc. ICDE, 2001, pp. 421-430.

[3] C.-Y. Chan, H. V. Jagadish, K.-L. Tan, A. K. H. Tung, and Z. Zhang, "Finding k-dominant skylines in high dimensional space," in ACM SIGMOD 2006, pp. 503-14.

[4] X. Lin, Y. Yuan, Q. Zhang, and Y. Zhang, "Selecting stars: The k most representative skyline operator," in Proc. ICDE, 2007, pp. 86-95.

[5] M. E. Khalefa, M. F. Mokbel, and J. J. Levandoski, "Skyline query processing for incomplete data," Proc. ICDE, pp. 556-565, 2008.

[6] J. Pei, B. Jiang, X. Lin, and Y. Yuan, "Probabilistic skylines on uncertain data," in VLDB 2007, pp. 15-26.

[7] E. Hüllermeier, I. Vladimirskiy, B. Prados Suárez, and E. Stauch, "Supporting case-based retrieval by similarity skylines: Basic concepts and extensions," in Proc. ECCBR'08, 2008, pp. 240-254.

[8] M. Goncalves and L. Tineo, "Fuzzy dominance skyline queries," in Proc. DEXA, 2007, pp. 469-478.

[9] A. K. Dey, "Understanding and using context," Personal Ubiquitous Comput., vol. 5, pp. 4-7, 2001.

[10] T. Strang and C. Linnhoff-Popien, "A context modeling survey," in Workshop on Advanced Context Modelling, Reas. and Manag., 2004.

[11] S. Holland and W. Kießling, "Situated preferences and preference repositories for personalized database applications," Conceptual Modeling, pp. 511-523, 2004.

[12] A. van Bunningen, "Context aware querying - challenges for data management in ambient intelligence," Tec. Rep., Enschede, Dec. 2004.

[13] K. Stefanidis, E. Pitoura, and P. Vassiliadis, "Adding context to preferences," in ICDE, 2007, pp. 846-855.

[14] A. Hadjali, A. Mokhtari, and O. Pivert, "A Fuzzy-RuleBased Approach to Contextual Preference Queries," in Proc. IPMU, 2010, pp. 532-541.

[15] Dubois and Prade, Possibility Theory. Plenum 1988.

[16] D. Sacharidis, A. Arvanitis, and T. Sellis, "Probabilistic contextual skylines," in ICDE, 2010, pp. 273-284.

[17] H. Prade, "Refinement of Minimum-Based Oredering in between Discrimin and Leximin," in Proc. Linz. Seminar on Fuzzy Set Theory, 2001, pp. 39-43.

[18] D. Dubois, H. Prade, F. Esteva, P. Garcia, L. Godo, and R. de Màntaras, "Fuzzy set modelling in case-based reasoning," Int. J. Int. Sys., vol. 13, pp. 345-373, 1998.

[19] P. Bosc, O. Pivert, and H. Prade, "A possibilistic logic view of preference queries to an uncertain data base," in Proc. Fuzz-IEEE, 2010, pp. 379-384. 